In reply to queries, Dr. Talbot said that in disease of the antrum the dentist should take entire charge of the case, which he should be competent to treat. I do not consider a man a real dentist who can not do so, though I fear there are comparatively few who are able to treat such cases successfully. I would not attempt to open into the antrum without knowing that it was necessary. I might go so far as to extract a tooth, but should then wait two or three months, if there was any doubt, to give time for a cure of the trouble by nature's help. Then, when sure that it was required, I should make the opening. In mild cases of antral trouble where transmitted light gives no indication I should treat the patient constitutionally, keeping the bow. els open and building up with tonics. Ninety-nine out of one hundred such cases are due to inflammation of the mucous membrane, as from the effects of grip or the inhalation of extremely cold air.

DR. C. S. LANE, Oakland-Is the drainage tube a necessity in cases where the antrum is opened? Will it not take care of itself without this aid?

Dr. TALBOT-The mucous membrane heals up very quickly if allowed to,-sometimes in twenty-four hours.

DR. I.ANE-Some surgeons say that it never heals, and my experience is that unless we do something to assist it, it does not readily heal. The idea advanced by Dr. Talbot seems new and well worth thinking of

Dr. DEAx-By this tube which Dr. Talbot shows, we have practically a valve which prevents the ingress of débris from the mouth. I do not feel able to discuss this paper to-day. Ask me a year from now and $I$ may be able to say something upon it.

\section{PYORRHEA ALVEOLARIS.}

Read in the Section on Dental and Oral Surgery at the Forty fith Annual Meetiug of the American Medical Association, held

BY W. J. YOUNGER, M.D.

$$
\text { SAN FRAXCISCO. }
$$

By this name, I mean that condition in which there exist patches of a slate-colored calculus partially or entirely encrusting the roots of the teeth, with the alveolus more or less destroyed and the surrounding gums deep red, retracted and congested - though sometimes attenuated; usually yielding pus at the cervical margin between it and the tooth, but this feature is sometimes absent.

This incrustation is firmly adherent to the cementum, for no pericemental tissue is ever found between it and the cementum. When removed it comes off in scales, leaving the portions of root from which it is detached, clean and white. This is true of the calculus on the shaft of the root, but that which is found around the apex of the fang is very difficult of removal, as it does not scale but has to be dragged off piecemeal or dissolved by chemicals.

This is not in consequence of any difference in the character of the tartar, but in the fact that the apices of the roots are not smooth like the sides and present a roughened surface and therefore a more secure attachment to the calculus.

In consequence of the waste of the bony walls of the socket, when this disease has progressed to any extent the teeth will be found loose and tender on pressure and frequently raised in their sockets or forced out of their position-usually towards the side opposite to the location of the tartar. If the disease is allowed to progress to a conclusion, the teeth will drop, or fall out, in consequence of their attachment to the alveoli being entirely destroyed.

The etiology of this disease is as yet surrounded in inystery, though the professional opinion is strongly drifting into regarding it as of constitutional origin; and the deposit of calculus (which is the bave of the disease and always present, some writers to the contrary notwithstanding) as being of hemorrhagic ori- gin, due to imperfect nutrition and connected with a gouty diathesis.

That it may be connected with malnutrition may be true, for no organ or tissue can become diseased while it is properly nourished; or that the condition may be aggravated or modified by constitutional disturbance or cachectic state is also palpable; but sufficient proof has not yet been adduced to establish the theory that pyorrhea alveolaris is of constitutional development and especially due to a gouty diathesis.

On the contrary, my experience with this disease (covering a period of over thirty years) has convinced me that it is of purely local origin; and I am very much inclined to the opinion I promulgated some ten or twelve years ago at a session of the California State Dental Association, that it is due to a diseased activity of the pericementum caused by disturbed nutrition or a local irritation, and that instead of bone it produces this disorganized abortion, vulgarly called tartar; itself perishing in the effort by the irritation of its presence.

This is the reason why pericementum is never found under the calculus. And this theory is strengthened by the fact that the constituents of tartar are analogous to that of bone, and why the pericementum is never in contact with the margin of the calculus. For there is always a denuded strip of root between it and the membrane.

While the irritation inducing the formation of this calculus may proceed sometimes from or be caused by the blood (as for instance the impaction of a capillary thrombus) I think it usually due to some irritant acquired from without. For the deposit is first formed near the cervices of the teeth, most frequently upon the approximal surfaces under the lingual margin where food and seed, etc., are most apt to lodge.

It is only in mature life and advanced age that we find it near the ends of the fangs; but in young or old one thing is certain; that between the alveolus and the root there is always free communication between the calculus and the cavity of the mouth. This condition, which in my long experience I have found invariable, shows the probability of some migrating germ or substance from the cavity of the mouth heing the original or exciting cause of the mischief.

Now were this disease of constitutional origin there certainly would be many cases in which there would be no lesion whatever between the gum tissue and the neck of the root. The pus seeking egress would pierce the alveolus and the gum just as in alveolar abscess. This would at least go to show that pyorrhea is not always due to an extraneous irritant. But even then it would not prove a constitutional origin and my theory concerning a diseased activity of the pericemental membrane and the lodgment of a capillary thrombus would be a better explanation. Moreover, I have found this disease to exist in an advanced condition, in persons who had always been exempt from any constitutional ailment whatever; who had no gouty or rheumatic diathesis either by inheritance or acquirement (at least had never developed any,) who never had indigestion or any form of dyspepsia, and who had (to use their individual expression, "the stomach of an ostrich" and had never been sick a day in their lives.

It was the consideration of such cases that made me once call pyorrhea alveolaris a "disease of good 
health." Another thing that tends to prove the disease is local is the fact that when once it is cured and the gums are made to re-attach themselves the disease does not return to them, no matter what cachectic condition may exist in the system.

As uric acid exists in the blood of the gouty, it is not to be wondered at that in persons of this diathe. sis, traces of the urates should be found commingled with the calculus of pyorrhea; for anv disturbing cause, such as tartar, that induces excitation and flow of blood to a part will almost of necessity acquire a deposit of whatever the blood is freighted with; and therefore the uric acid which Professor Peirce found in some of the calculus and upon which he based his assumption, that the calculus was the result of a gouty condition, was simply the natural outcome of the presence of an over-supply of blood, and had nothing to do with the origin of the calculus.

But the best way to try a theory is to test it clinically; and if, for instance, in pyorrhea alveolaris it is found that the disease can be prevented or cured by constitutional treatment, then we must regard that disease as of constitutional origin. But if, on the other hand, you find a local disturbance or lesion yielding to local treatment, solely, and remaining cured regardless of any systemic pathologic condition, then you may reasonably conclude that this disease is local. And from my experience with pyorrhea alveolaris, I would as soon think it of constitutional origin, as I would that corns, bunions and freckles are of systemic development.

And now to consider some cases in point:

A lady who had a decidedly gouty diathesis, through inheritance (so marked that her finger joints became tender and swollen and the deposit of uric salts was being continually formed in the follicles of her throat) went to a friend of mine, one of the cleverest men in the profession, to have three upper molars, two on the left and one on the right in nearly the last stages of pyorrhea alveolaris, treated and filled. Their roots were so thoroughly incrusted with tar tar that they merely hung in their sockets by their apical extremities, and they were all more or less decayed, but especially the right molar, for it had a cavity extending almost to the pulp. She had also suffered for two or three years from frequent attacks of nervous prostration, and the impression commenced to grow that this condition was due, in a measure at least, to the state of her teeth, which in consequence of their looseness and tenderness made it impossible for her to masticate properly.

This gentleman advised her to have these molars drawn immediately for the sake of her health, as he considered it impossible to save them, but as the lady would have to substitute artificial dentures she demurred, and hoping to spur him on to some effort to preserve them she remarked: "I think Dr. Younger could save them!" "Madam," was the retort, "neither Dr. Younger nor God Almighty could save those teeth. They are so joosened by disease, I could pull them out with my fingers!"

The idea that God Almighty should not be able to save them grated on her religious sensibilities, and she deter mined to wait until my return. I was then on my way home from Berlin, and one of the first persons to consult me on my arrival was this same lady. Having made a spe cialty of just such cases, I commenced treating her,ignorant of the judgment that had been passed upon the teeth by my esteemed colleague. She feared to tell me until the cure was effected, lest it might prejudice my efforts. In six weeks the teeth were firm and the alveolar tissue strongly adherent throughout their full extent, certainly around the cervices.

It is now nearly four years since the cure was completed, and though the finger joints still swell and become tender and the uric incrustations still form in her throat, the teeth have had no return of the trouble; they are perfectly rigid in their sockets, and there is nothing in their appearance, nor in that of the environing gums, which would suggest the lesion which had been so nearly fatal to their existence. Now no constitutional treatment was made, the success being due entirely to local applications.

Some eight or nine years ago, at a meeting of the State Association, the subject of pyorrhea was in discussion and there was an almost unanimous opinion expressed that this disease could not be cured and that the cures I mentioned could not have baen "true pyorrhea." While I was on my feet arguing this, Dr. Cummings of this city came to me, saying: "Can you cure me?" and opened wide his jaws. His teeth were all loose, the swollen gums had retracted from around the roots for an eighth of an inch, and pus was flowing at the slightest touch. I replied: "Yes, if you will permit me to clinic on you before these gentlemen, and prove to them how wrong they are." I then called the attention of the convention to the condition of Dr. Cummings' mouth and asked them to tell me what the matter was. With one accord, they said, "pyorrhea." "Bad case?" "Yes, a very bad case." "Now, gentlemen," I said; "the proof of the pudding is in the eating. I will treat Dr. Cummings before you, and at our next session you will see whether pyorrhea can be cured or not."

I gave the Doctor half a dozen treatments, all local, and the disease quickly yielded; and though eight or nine years have passed since then, there has been no return of the disease, and the teeth and gums are firm and sound.

A few years ago, I commenced treating a gentleman of mature years for pyorrhea. He was also being treated for gout. The pyorrhea was on a fair way to cure, when one fine morning he told me that his physician had informed him that my local treatment could not possibly save his teeth ; that the disease in his gums was the result of gout, and had to be treated constitutionally. He therefore dropped me and stuck to his physician; who was a very learned man with a microscope. The result was he was relieved of his gout, also of his teeth, for under the "constitutional treatment" they all dropped out! Had he stuck to me, alone, he would have had his gout, but he would also have had his teeth. He should have stayed by both of us, and now he would have his teeth and be minus his gout.

It is a fortunate circumstance that a knowledge of the etiology of pyorrhea alveolaris is not a necessary factor for its cure. There are few lesions in surgical pathology simpler of treatment than this same disease; the most essential requisite being skill and manipulative dexterity, in order to secure the thorough removal of the calculus and the perfect cleansing of the root. The elimination of every particle of tartar is a sine qua non to success. Unless this is done perfectly and absolutely, the disturbing cause of the disease will not be eradicated and it will in time return.

The return of the disease that so many complain of is due very often to the fact that the complete removal of the tartar has not been accomplished, and that the surface of the roots has not been thoroughly cleansed. The appreciation of tartar, however, is not always easy and it requires a certain sensibility in the fingers not common to all persons.

It is that touch which makes one violinist superior to another in the development of the tone of his instrument. This is the reason why so many have failed to discover tartar when it existed, and why it has led them to suppose that either the tartar had been completely removed or that the pyorrhea existed without the tartar. There is, besides the sense of 
feeling, an acuteness of hearing by which one can recognize by sound, as the instrument passes over the root, whether it is the substance of the root or an incrustation.

But before the removal of the tartar is attempted, the mouth and crevices and the little pockets containing the tartar and the calculus itself, should be thoroughly sterilized. I prefer the bichlorid of mercury, 1 to 1000 . It should be used warm, at least that portion that is injected around the necks of the sensitive teeth, in order to prevent the pain that cold applications are almost certain to produce. The sterilization is necessary to prevent the absorption of septic matter, as the gum is almost certain to be abraded by the instruments used to remove the tartar. For the same reason the instruments should be thoroughly sterilized. The water used as the menstruum for the sterilizers should have been subjected to distillation; otherwise the organic matter and chemicals which are apt to be in ordinary water may be sufficient to decompose or to modify the strength of the sterilizing agent and so negative its action. But, by all means, be sure and sterilize the instru. ments! Keep them continually in the antiseptic fuid while laid out for use.

When removing the calculus, begin with one tooth and stay by it, until all of the tartar is removed, even if it takes the full time of the sitting. And as fast as the tartar is scaled off, wash it out with the warm fluid by syringing with delicate platinum pointed syringes. If this is not done, minute pieces of the tartar will adhere to the raw surface of the gum, keep up an irritation, and prevent the healing process from being established. It is well, when the pookets are very deep, to insert a little lactic acid to dissolve the diseased edge of the alveolus and any minute fragment of tartar which may have escaped the instrument.

The root being thoroughly cleansed, and the débris washed out of the pocket, a little tincture of iodin may be dropped into it, or else a solution of chlorid of zinc, three and one-half drachms to the pint of water, to stimulate its contraction and the production of granulations, and to induce the attachment of the alveolar tissue and gums to the root. If this does not take place, then rub quickly with a little wad of cotton saturated with liquor ammonia, washing out immediately with warm water. This ammonia is to remove the mucous membrane from the healed and therefore unattachable surface of the gums; and the water, to wash it off before its escharotic action can be established. By this means a healthy granulating surface is formed, contraction stimulated, and attachment to the clean surface of the root induced. In case the gum should remain flabby, inactive and indoleut, then acupuncture should be resorted to This can be done by rapidly piercing the gum with a sterilized needle. When the amount of the deposit is so great that the alveolar septi have been destroyed, pass a threaded needle through the gums and between the roots of the teeth and approximate them by tying them as nearly together as can be done, without the use of much force. If too much force is used the thread will cut through the gums.

In the case of one lady of 55 , the accumulation of tartar was so great that the septi of both gum and alveoli from the first bicuspid of one side to the first of the other were destrosed, and the labial and lingual walls of gums simply leaned against the teeth when the mouth was in repose, but left them instantly when the tongue or lips moved. For instance, when the lower lip was pushed down, all the wall of the gum upon its aspect went forward with it, leaving the roots of the teeth entirely denuded of gum upon their front. When the tongue was raised or retracted, the gum upon that side moved away also, leaving bare the teeth upon the lingual surface. And when the tartar was removed the separated teeth leaned in every direction.

I gave the gums the ammonia treatment; held them in position by means of ligatures through the interdental spaces; kept the mouth in an aseptic condition by the repeated use of the sublimate, every two hours. In a couple of weeks I had the gratification to see the spaces filled with granulations as high as the margins and firmly binding the two layers of gum into one continuous mass.

This case was reported to the State Dental Association and to the Surgical Section of the Ninth International Medical Congress in Berlin.

The reason for treating the teeth one by one-that is, completing the removal of the tartar and all that has to be done, at one sitting, is that the process of healing should not be interfered with after it has commenced, and also because there is the object of getting, so far as possible, the benefit of healing by first intention.

By the subsequent use of antiseptic washes, until the healing process was entirely accomplished, I have succeeded in curing the most desperate cases of pyorrhea alveolaris, and of restoring to perfect use, teeth that have been for years worse than worthless.

There is only one condition in which I have found failure, and that is where there is a dead pulp in connection with an incrusted apex. This condition is found almost exclusively in the palatal root of the superior molar, and the difficulty in the way of a cure is due, not so much to the tenacity or quality of the tartar, as it is to the septic condition of the substance of the fang, the putrescence of the pulp having invaded tubuli and canaliculi and destroyed beyond hope of restoration the vitality of its tissue, and thereby its ability to re-form attachment.

As the diseased pulp is usually confined to the palatal root-that in the buccal being usually healthy-my treatment in these cases has been to open into these roots, remove their pulps, fill them and amputate the palatal; then grind away enough of the articulating surface of the crown, immediately over the removed root, in order to bring the pressure in the effort of mastication upon the buccal roots. By these means, these teeth can be made comfortable and serviceable for years, if not for a lifetime.

In eleven cases of pyorrhea reported by Dr. C. N. Peirce and Dr. Albert Brubaker, wherein they sought to establish their theory that uric salts were found in the incrustations of what they call "true pyorrhea," in but five of these eleven cases were they able to discover traces of uric salts. Now, although exceptions are said to prove the rule, where the exceptions are more numerous than the rule sought to be established, the exceptions govern the rule. Therefore, the cases decided by Drs. Peirce and Brubaker are not sufficient to establish a theory on which to base their assumption and their conclusion.

There can be no local lesion or irritation which is not more or less affected by the state of the system, that when vitiated will prevent the healing of an otherwise simple sore which under normal conditions would yield readily to local applications.

Dr. Brubaker makes a statement that the deposit of uric salts upon the end of the roots is being "continually renewed." One would infer from the term 
"renewed" that the deposit is absorbed and more salt around the roots, and the discharge of pus, are modre-deposited; but as such a statement would be ified by constitutional treatment.

absurd he can not possibly mean that. The only inference to be drawn then, is that if the deposit is removed by instruments or chemicals, a fresh deposit of the uric salts would take place, and thus a source of irritation would be continually kept up. Now both Drs. Peirce and Brubaker claim that this condition only takes place in the apex of the fang; but why should it be limited to the apex of the fang? The same exact tissue surrounds the sides of the roots as that which envelops the end. Then why limit this particular action to this particular locality?

The fact is that, when a deposit incrusts the entire end of a root, the irritation set up by its presence results in the death of the pulp, and this condition with the greater vascular activity set up by the propinquity of the calculus to the larger blood vessels entering the foramen of the apex causes in a greater degree a deposit of any salt the blood is freighted with. Therefore, in the few cases in which an examination revealed the presence of the urates such a presence was but a natural conclusion, and the only wonder is that the salts were not found in the entire eleven cases cited instead of only in five.

I do not know why it is that Dr. Peirce calls that condition, only, where the calcic formation surrounds the apex of a root, "true pyorrhea alveolaris." This condition exists so rarely when compared with what he calls ptyalogenic calcic pericementitis (but what is known to the profession as pyorrhea alveolaris) that it reminds one of a fanciful nume given by early settlers to a certain region in this State, "Strawberry Valley," for the reason that there were no strawberries in the valley! If he wished to make a distinction between the calcic formation on the shaft of the fang and that of the apex he should have left that general condition known as the "true pyorrhea" and given a name to the special condition.

But why is it that this special condition exists almost, if not entirely, on the palatal root of the first superior molar? It is easy to be seen from the fact that the palatal root of the first molar spreads more than that of the multi-rooted teeth. That the tissue over them is more attenuated than it is over the roots of the other teeth, and that they are therefore more affected by thermal changes than the other roots; that the pressure of the tongue and the superimposed food is greater upon these roots; in fact, that the impact of food is stronger on these roots than on any other. They are, therefore, more exposed to changes and vicissitudes than the buccal roots of the same teeth; or of the roots of any of the other teeth. That is the reason why we so often find these roots more denuded and exposed than the other roots, and why the pulps in these roots are more susceptible, in consequence, to abnormal changes and death.

In concluding this paper, perhaps it would be well for me to summarize the arguments for and against the theory of pyorrhea alveolaris being due to constitutional diathesis. The arguments in favor of pyorrhea alveolaris being of constitutional origin are what?

1. That it is found in persons afflicted with gouty diathesis.

2. That traces of uric salts are found in the calculi of some of the people afflicted with the gouty diathesis.

3. That the condition of irritation in the gums and

In opposition to this we find :

1. That pyorrhea is found in persons having no constitutional cachexia or diathesis.

2. That if it were of constitutional origin it would be found in all, or at least in nearly all cases having the gouty disposition.

3. That the amelioration of the irritation by constitutional treatment is what would occur in any local irritation when the system would be placed in a healthy condition.

4. That if it were of gouty diathesis the deposits from the saliva would also be impregnated with these urates.

5 . That, if it were of constitutional origin, constitutional treatment would be necessary for its cure; but, instead, local treatment is found sufficient for its complete eradication, notwithstanding the constitutional ailment is continued in undiminished force.

6. In all cases of pyorrhea alveolaris we find a connection between the calculus and the cavity of the mouth, which would not necessarily be so if it were the result of a constitutional pathologic state.

Besides these points, Dr.Van Woert asserts that the disease is communicable by infected instruments which, if true, would prove a very strong argument against the theory of constitutional development.

\section{DISCUSSION.}

Dr. C. S. LANE, Oakland-These papers are of great interest to those of us who are in active practice, meeting with cases of the characters described every day, and studying to overcome the conditions as we find them. The difference in the positions taken by the writers is an evidence that great minds do not always see things in the same light. One thing that strikes me practically in this disease, is that in some cases I can overcome the condition by local treatment; by a thorough removal of the deposit the way is paved for a thorough cure. In some instances the cure is not so thorough. I have always striven to be very careful in the local treatment of this disease, and yet I find some cases where I can not put a stop to the suppuration, more especially in diabetic patients. I have now one case under treatment which has been in my hands over three years; I have carefully watched the reported discussions on this disease by the leading practitioners, and though I have tried everything that has been suggested I have been unable to arrest the progress of the disease; so that I can not think in this case the cause can be considered due to local conditions, or that its persistence is due to any lack of proper sanitary conditions or local surgical treatment. Many cases of pyorrhea alveolaris can be treated locally successfully, but there are others, especially among patients with diabetic troubles, for the cure of which you have to go farther than merely local treatment.

Dr. YounGER-A case which occurred lately in my practice is called to mind by the remarks of Dr. Lane. A lady, in an anemic state, presented just before an expected six weeks' banishment from the pleasures of the world, with pockets discharging pus around several of the teeth. On careful examination a slight rim of tartar was found near the alveolar process, the disease having progressed on one of the teeth to within an eighth of an inch of the apex. This tooth was so loose that every breath waved it to and fro. I spent one and one-half hours on each tooth, carefully removing every trace of the deposit, and the disease was cured. I used iodin in the treatment. One thing to be borne in mind in the local treatment of these cases is that plenty of time must be taken to each tooth; otherwise you will fail. I think that in the disinclination to do this is the cause of failure frequently. Then again you must have the natural touch that will enable you to know absolutely when the removal of the deposit is accomplished. I know of no other reason why I am successful in the treatment of these cases than the possession of this touch and the taking of the necessary time to do the work thoroughly. You all know me, and you know that I am honest in the opinions I express, and that I take the ground I do, only 
because I believe it will be for the benefit of the profes. sion. One reason why I have given the clinical treatment of pyorrhea alveolaris in detail is because I believe the cure is to be found in the careful manipulation of the instrument point, rather than in high scientific theories. I have also received letters from different places, among them Bombay and Krakow, asking how I treat pyorrhea, and this seemed a fitting opportunity to give a general reply.

Dr. Joux C. McCoy, Santa Ana-How do you keep patients still for one and one-half hours?

Dr. YoCNGER-By not hurting them. I obtund the sensibility of the tissues so as to cause only the minimum of pain.

Dr. Russeli H. Cool, Oakland-I have seen Dr. Younger operate, and his fingers are certainly sensitive. As he says, the proper removal of the deposit in pyorrhea alveolaris re quires delicacy of touch; the deposits are tenacious, and you must take whatever time is necessary to remove them. thought it impossible years ago to cure this disease by local treatment alone, but I have seen cases so cured by Dr. Younger. He mentions warm water in his description of the treatment. He should have said hot-at least as warm as the patient can bear, in connection with the bichlorid. Pyorrhea does not spring up in a night, and I have noticed in young patients, especially, you will find a single pocket, to dislodge the deposit from which requires but little pushing. If neglected, however, we find it spread from this incipiency till it involves several teeth. The successful man is he who treats the disease in its incipiency without waiting till diabetes is established and the man is on the verge of the grave. You sometimes find in these cases a little abscessed cavity along the course of the pocket. A surgical opening will give room to medicate and treat this.

Dr. I. N. Demarest, Santiago, Chili, was here introduced to the Section, but he excused himself from making any rema'rks ).

Dr. G. S. Dean, San Francisco-I would like to ask a question. Dr. Younger and Cool, and perhaps Dr. Dunbar also, have referred to the occurrence of an abscess between the gingival border and the apical space. Do they mean an actual abscess?

Dr. R. H. Cool-I meant a sinus containing pus. In one case which I recall at the moment, there was such a sinus on the labial surface of one of the teeth. By cutting through the alveolar plate, I opened into it and then treated the same as any other abscess.

Dr. Dean-I want to call for more testimony. Abscesses which are not apical, we may perhaps call gingival abscesses.

Dr. Ergene S. Talbot, Chicago-We have heard both sides of this question-the local and the general-in the papers which have been read, neither of which. however, classified the various forms of pyorrhea alveolaris. I have certainly seen three different conditions which properly come under this name. First, there is the local deposit from the salivary glands; then the serumal deposit directly from the capillaries; and last, a condition which I must say is constitutional, because there is no deposit whatever. I can't har monize this last with Dr. Younger's conclusions; I don't care if he don't hurt the patient, and no matter if he does take one and one-half hours to each tooth. Take a case of serumal tartar. If Dr. Younger can remove all the deposit, I can not. I don't even know when it is all removed. Take a mouth in which there is a set of good sound teeth, but in which this disease has affected the second molar to within one-eighth of an inch of the apex, the tooth being in consequence quite loose, while others are just beginning to become loose, from the effects of serumal tartar. We will clean that loose tooth thoroughly. We find the ends of the roots exposed and the tooth itself held in the process by one root only. We clean the teeth as well as we can, and in one month's time the patient comes back with the tartar ex tended all around the mouth. If we remove that loose tooth, treat as before, and watch the case, we can save the other teeth; but as long as that loose tooth remains in the mouth it forms a nidus or rallying place for the destructive forces. I have had that experience over and over again. In the third condition to which I have referred there is no deposit. I have seen cases of this kind where the teeth were loose, and I tell patients from the start that nothing can be done by local measures, and I simply treat them constitutionally. They become built up and the trouble abates; then they stay away for three months and come back all run down with the old condition renewed. I have had other cases where the condition was inherited-not the pyorrhea but a predisposing condition of the system which was handed down from generation to generation. As long as we practice dentistry as we do to-day, we give evidence that we don't know the principles underlying this disease. We have to get a better knowledge of it. I believe pyorrhea alveolaris is a constitutional disorder, and that it is not due to uric acid in the system, but that the liver is the cause of it and we have to get down to treating that before we can master this disease. It arises from an inherited condition of the liver and not until we know more about the treatment of the liver than we now do shall we know how to really cure pyorrhea.

Dr. YOUNGER-Dr. Talbot asserts that we are as ignorant about pyorrhea alveolaris to-day as we were twenty-five years ago. I would like to know what is known in medicine about for instance, the function of the spleen. I would like to know what the medical profession has taught us about the origin of disease. I think we do know a great deal more about pyorrhea than we did, and that we are able to treat it better.

Dr. BonwILL-I don't like to repeat here what I have just said before the Odontological Society of Pennsylvania where the subject was recently discussed. The majority of dentists don't know how to treat pyorrhea alveolaris. I stated my ideas two years ago in combating the idea of a calcic diathesis being the cause. I agree with Dr. Younger in everything he says, but I go a step further than he does. Dr. Younger looks at the matter in a common sense light. Nothing shows more plainly the uselessness of the dental profession as an appendage to the medical than this one subject. Dr. Younger sees a phenomenon-tartar on the roots of the teeth, which is the true cause of pyorrhea. I have had forty years' experience in the practice of dentistry, and I should be ashamed to have a case of pyorrhea in a mouth of which I had entire control, because I would say it was my fault. In forty years' practice I have not a single case of a regular patient of mine that had pyorrhea. There must be perfect cleanliness. Dentists too often neglect this first important requisite of cleansing the teath and keeping them clean. The first thing to do before daring to touch a tooth to operate upon it is to see that the mouth is clean. Half of $\mathrm{my}$ cases of this disease are the failures of other men. I recall at the moment the case of a patient who came to me after he had been to a number of medical men who told him he would lose all his teeth. Three of them were in fact so far gone that they had to be extracted, but the others were saved. If you cleanse these teeth one by one, take away all the dead tissue the same as Dr. Younger does, cut off the superabundant gum, and cleanse thoroughly they will get well. I have never yet in forty years given a patient constitutional treatment for this trouble. If, after having put the teeth in proper condition you teacb patients proper habits with regard to their teeth, there will be no return of the disease. I cleanse the whole upper set if it is at all affected, at once, even if it takes the whole day; cut out the superabundant gum, and don't meddle with nature after giving her a chance to do the work of repair. The gums will again hug up to the teeth. For treatment, there is nothing better than creasote and I now use carbolic acid and not diluted. I attribute much of the cause of pyorrhea to bad dentistry. In almost every mouth under the regular care of the dentist you find that teeth have been extracted in early life; or that that they are cut apart on the approximal surface and filled with flat filling, cutting away the gum to make room. Overhanging fillings at the cervix, amalgam fillings that their author ought to be ashamed of, crude and rough as they are-will destroy the membranes around any tooth. Gold crowns which are not fitted properly or finished fine at the edges are also factors in this disease. Then the rubber dam must be tied on or held up beyond the gum margin, and is another cause of trouble at the cervix. There are many other causes which may help tooriginate this disease, but those I have mentioned are enough to give my ideas. I am a crank on articulation, for instance. If there is anything which helps out in these cases it is seeing that the articulation is correct. I always take an impression and then knowing the law of articulation, with a little study I can see just where to touch the teeth and correct any deficiency. I am against the principle of the so-called gouty diathesis, and in treating teeth I never cut away too much, but rather too little.

Blank Applications for membership in the Association, at the Journal office. 